\title{
Relationship between the rewarding and aversive effects of morphine and amphetamine in individual subjects
}

\author{
Andrey Verendeev • Anthony L. Riley
}

Published online: 28 June 2011

(C) Psychonomic Society, Inc. 2011

\begin{abstract}
Drugs of abuse have been reported to produce both rewarding and aversive effects, as evidenced by their ability to induce both conditioned place preferences (CPPs) and conditioned taste aversions (CTAs), respectively. Although several attempts have been made to assess the relationship between the rewarding and aversive effects of drugs in independent groups, it is unknown to what extent (if any) preferences and aversions are related in individual animals. The present study assessed this relationship by examining the ability of morphine ( 5 and $10 \mathrm{mg} / \mathrm{kg}$ ) and amphetamine ( 3 and $5 \mathrm{mg} / \mathrm{kg}$ ) to induce both place preferences and taste aversions in the same animal, using a concurrent CTA/CPP design. There was no consistent relationship between the ability of morphine or amphetamine at either dose to increase time spent on the drug-paired side and the ability to suppress consumption of the drug-paired taste. These results support the position that drugs of abuse have multiple stimulus effects, both rewarding and aversive, that condition place preferences and taste aversions independently.
\end{abstract}

Keywords Morphine - Amphetamine - Conditioned taste aversion $\cdot$ Conditioned place preference $\cdot$ Concurrent assessment $\cdot$ Individual differences

A. Verendeev $(\bowtie) \cdot A$. L. Riley

Psychopharmacology Laboratory, Department of Psychology,

American University,

Washington, DC 20016, USA

e-mail: andrey.verendeev@gmail.com

\section{Introduction}

Drugs of abuse have been reported to produce both rewarding and aversive effects, as evidenced by their ability to induce both conditioned place preferences (CPPs; see Tzschentke, 2007, for a comprehensive review of CPP) and conditioned taste aversions (CTAs; see Riley, Davis, \& Roma, 2009; see also www.CTAlearning.com), respectively. This ability has been discussed as a paradox by many (see Gamzu, 1977; Hunt \& Amit, 1987; White, Sklar, \& Amit, 1977; Wise, Yokel, \& DeWit, 1976), as well as a basis for suggesting common underlying mechanisms for reward and aversion learning by others (see Grigson, 1997; Hunt \& Amit, 1987).

Two features are important to note in discussing the work on drug-induced CPPs and CTAs. First, assessments (and comparisons) of the rewarding and aversive effects of drugs of abuse are generally made in different groups of subjects (and often in different studies). Second, demonstrations of these rewarding and aversive effects are generally based on group means, with no analysis of individual-subject data. When examined together, these two features pose a potential concern for conclusions regarding the dual effects of drugs of abuse and their relationship to each other. Specifically, they preclude a determination of the relationship of reward and aversion in individual subjects. That is, from such comparisons, one cannot determine whether an animal that acquires a strong place preference (relative to controls and other drug-treated subjects) would or would not display a comparably strong taste aversion (and vice versa). Given that there is considerable individual variability in these behaviors (see Allen, Everett, Nelson, Gulley, \& Zahniser, 2007; Farber, 
Gorman, \& Reid, 1976; Riley, Jacobs, \& LoLordo, 1978; $\mathrm{Xu}$, Wang, Wu, \& Pei, 2001), group analyses may indicate little about the actual relationship between CPPs and CTAs.

One attempt to assess the relationship between reward and aversion in the same subject was reported in a study by Turenne and her colleagues (Turenne, Miles, Parker, \& Siegel, 1996). In this report, Turenne et al. first gave rats access to saccharin, followed by an injection of morphine or amphetamine (taste aversion conditioning). The individual differences in the magnitude of the CTA were then assessed, and the animals were assigned to high-CTA or low-CTA groups on the basis of their aversions to the saccharin solution. These subjects were then injected with morphine or amphetamine and placed on one side of a CPP apparatus in an attempt to establish a CPP to the drug-paired side. The authors reported that the group that displayed the strongest amphetamine-induced CTA also displayed the strongest amphetamine-induced CPP; however, for the morphine-treated subjects, there was no significant difference in place preferences for animals in the high- and low-CTA groups.

Although Turenne et al. (1996) examined the rewarding and aversive effects of morphine or amphetamine in the same subject, theirs was nonetheless a group analysis in which the presence or absence of an effect was based on group means. There was no determination of the relationship between place preferences and taste aversions in individual subjects. A more appropriate assessment of the relationship between the rewarding and aversive effects of drugs would be to examine these effects concurrently in the same animal. In such a design, individual animals would be given a novel solution to drink, injected with an aversioninducing drug such as morphine, and then placed in a distinct chamber. On subsequent tests, the avoidance of the drug-associated taste and preference for the drug-associated chamber would index the drug's aversive and rewarding effects, respectively. Although not commonly used in assessments of the affective properties of drugs, this design has characterized the rewarding and aversive effects of several compounds - for example, caffeine (Brockwell, Eikelboom, \& Beninger, 1991), morphine (Martin, Bechara, \& van der Kooy, 1988; Sherman, Pickman, Rice, Liebeskind, \& Holman, 1980; Simpson \& Riley, 2005), and amphetamine (Reicher \& Holman, 1977; see also Switzman, Amit, White, \& Fishman, 1978, who used a concurrent runway/CTA procedure). Interestingly, in none of these assessments was the focus on individual subjects. Consequently, no individual-subject data were reported to allow an analysis of the relationship between reward and aversion.

The present series of studies used the concurrent design described above and examined the relationship between CTAs and CPPs in individual subjects. Specifically, rats were given a novel saccharin solution to drink, injected with morphine $(5 \mathrm{mg} / \mathrm{kg}$, Experiment $1 ; 10 \mathrm{mg} / \mathrm{kg}$, Experiment 2$)$ or amphetamine $(3 \mathrm{mg} / \mathrm{kg}$, Experiment 3 ; $5 \mathrm{mg} / \mathrm{kg}$, Experiment 4) and immediately placed on the nonpreferred side of a place preference apparatus. All subjects received one-trial conditioning in order to avoid any floor or ceiling effects (i.e., complete suppression of consumption or asymptotic preferences) that would limit sufficient individual variability for meaningful correlational analyses. The drugs - that is, amphetamine and morphineused in the present series of assessments were chosen to match those used by Turenne et al. (1996). The doses of amphetamine ( 3 and $5 \mathrm{mg} / \mathrm{kg}$ ) were similar to those used by Turenne et al.; whereas Turenne et al. used a single high dose of morphine $(15 \mathrm{mg} / \mathrm{kg})$, the present analyses used two lower doses $(5$ and $10 \mathrm{mg} / \mathrm{kg}$ ) that have been reported to condition intermediate levels of taste aversions (RandallThompson \& Riley, 2003; Simpson \& Riley, 2005).

The assessment of the relationship between the rewarding and aversive effects of drugs of abuse may further our understanding of the nature of the underlying mechanisms that mediate these effects. For example, Grigson (1997) has argued that a drug's rewarding effects mediate suppression of saccharin consumption within the CTA preparation. According to this position, there should be a significant and direct relationship between the ability of the drug to condition a place preference and a taste aversion in an individual animal. The present series of experiments allows for a direct assessment of this hypothesis and others assuming a common mechanism (see also Hunt \& Amit, 1987). If, on the other hand, these opposing motivational effects are mediated by different mechanisms, no relationship between drug reward and drug aversion would be expected. If preferences and aversions do not covary, it would suggest that these are two independent effects mediated by different processes (Cunningham, Gremel, \& Groblewski, 2009; Parker, Limebeer, \& Rana, 2009).

\section{General method}

The following general method applied to each of the four experiments used in the assessment of the relationship between drug reward and aversion. The only difference among the four experiments was the specific compound (or dose) used during conditioning.

\section{Subjects and housing}

Subjects were 99 drug-naïve, male Sprague-Dawley rats. They were individually housed in hanging wire-mesh cages with ad libitum access to food and water and were maintained on a 12:12-h light:dark cycle (lights on at 
$0800 \mathrm{~h})$ and at an ambient temperature of $23^{\circ} \mathrm{C}$. Graduated $50-\mathrm{ml}$ Nalgene tubes were placed on the front of the cages for presentation of either water or saccharin (between 0800 and $1200 \mathrm{~h}$ ). All procedures were conducted under the guidelines established by the Institutional Animal Care and Use Committee at American University and were in compliance with the Guidelines for the Care and Use of Laboratory Animals (National Research Council, 1996).

\section{Apparatus}

The place conditioning apparatus (San Diego Instruments Place Preference System, San Diego, CA) consisted of two main conditioning chambers $(28 \times 21 \times 34.5 \mathrm{~cm})$ joined by a smaller middle chamber $(14 \times 21 \times 34.5 \mathrm{~cm})$. One of the conditioning chambers featured a white aluminum diamond plate floor with white walls; the other conditioning chamber featured a haircell-textured black plastic floor with black walls; the smaller middle chamber was outfitted with a steel rod floor and gray walls. Each individual chamber in each apparatus had its own white LED lights, and the lights were set on minimum. The CPP room was illuminated by a $25-\mathrm{W}$ red light mounted to the ceiling, and a white noise generator was used to mask background noise. A total of eight identical apparatuses were used; each apparatus featured a $16 \times 4$ photobeam array for recording time (in seconds) in each chamber.

\section{Drugs and solutions}

Both morphine sulfate and amphetamine sulfate (generously provided by NIDA) were prepared as a $5-\mathrm{mg} / \mathrm{ml}$ solution in physiological saline (drug vehicle). Morphine was administered subcutaneously, and amphetamine was administered intraperitoneally. Saccharin (0.1\% sodium saccharin, Sigma Chemical Co.) was prepared as a $1 \mathrm{~g} / \mathrm{l}$ solution in tap water.

\section{Procedure}

Habituation and CPP pretest Subjects were restricted to 20-min water access each day until body weights and fluid consumption stabilized and all subjects were approaching and drinking water from the tube within $2 \mathrm{~s}$ of its presentation. On the day before conditioning, each animal was allowed 15-min access to the entire place conditioning apparatus to obtain individual baseline times spent in each chamber. A paired samples $t$-test comparing time spent in the two chambers in each experiment revealed that subjects spent significantly more time in the black chamber than in the white chamber (all $t \mathrm{~s}>4.885$, all $p \mathrm{~s} \leq .05$ ). Given this initial bias, the drug was subsequently paired with the nonpreferred white chamber (drug-paired side[DPS]). Indi- vidual subjects that demonstrated an initial preference for the white chamber (100 s or more) were excluded $(1,2,5$, and 3 subjects from Experiments 1-4, respectively), resulting in 20-23 subjects for each experiment (see below).

Conditioning and CTA/CPP testing Subjects were run between 0800 and $1200 \mathrm{~h}$ to avoid robust aversion conditioning manifested when rats are conditioned in the dark phase of the light:dark cycle (Gomez-Serrano, Kearns, \& Riley, 2009). Eight subjects were run at any one time, and each session lasted $1 \mathrm{~h}$. This resulted in three runs per day. Both drug- and saline-injected subjects were included in each run. On the first conditioning day, animals in each group were given access to a novel saccharin solution in their home cages during their normal daily 20-min fluid access period. Five minutes after the removal of saccharin, the animals were injected with either a drug or equivolume saline and immediately placed in the white chamber (DPS) of the CPP apparatus for $30 \mathrm{~min}$. On the next day, animals received 20 -min access to water, followed by an injection of saline, and then were placed in the black chamber (NDPS). On the following day, all of the animals were given a test for CPP, during which the subjects were placed in the middle gray compartment and given 15-min access to the entire apparatus in a drug-free state. On the day following this preference test, all subjects were given a one-bottle aversion test in which they received 20-min access to the saccharin solution in the home cage.

\section{Statistical analyses}

For each experiment, place preferences were analyzed using a paired samples $t$-test comparing time spent on the DPS on the preference test to time spent on the DPS on the pretest (baseline level). Taste aversions were analyzed using a paired samples $t$-test comparing saccharin consumption on the aversion test with consumption on the initial exposure to saccharin (baseline consumption). All drug-injected subjects were then divided (median split) into high- and low-CTA groups ( $n=5-6$ each) on the basis of the strength of their taste aversions (i.e., change in amount of saccharin consumed), and their place preferences were analyzed. Similarly, subjects were divided (median split) into highand low-CPP groups ( $n=5-6$ each) on the basis of the strength of their place preferences (i.e., change in time spent on the DPS), and their taste aversions were analyzed. The relationship between changes in time spent on the DPS and changes in saccharin consumption was then determined for each experiment, using Pearson correlation coefficient. Statistical significance for all analyses was set at $\alpha=.05$. 


\section{Results}

Experiment 1: morphine, $5 \mathrm{mg} / \mathrm{kg}$

As was noted, individual subjects injected with morphine $(n=12)$ were divided into high $(n=6)$ and low $(n=6)$ responders for both the CTA and CPP preparations. Subjects in the high, $t(5)=-5.922, p<.01$, but not the low, $t(5)=-1.536, p>.05$, CTA group significantly suppressed their saccharin consumption, relative to their own baseline (Fig. 1, left panel). When the high- and lowCTA groups were separately analyzed for place preferences, neither the high, $t(5)=0.515, p>.05$, nor the low, $t(5)=2.022, p>.05$, CTA group displayed a significant increase in time spent on the DPS (18- and 146-s change from baseline preference, respectively). Correlational analysis of individual subjects revealed no significant relationship between changes in saccharin consumption and changes in time spent on the DPS for either of these two conditions, absolute $r \mathrm{~s} \leq .497, \mathrm{ps}>.05$ (see Table 1).

The high-CPP $(n=6)$ group, $t(5)=4.472, p<.01$, displayed a significant increase in time spent on the DPS, whereas the low-CPP $(n=6)$ group did not differ from its baseline, $t(5)=-1.168, p>.05$ (Fig. 1, right panel). When these groups were analyzed for taste aversions, both high-, $t(5)=-3.162, p<.05$, and low-, $t(5)=-2.739, p<.05$, CPP groups displayed a significant decrease in amount of saccharin consumed ( -2 and $-4-\mathrm{ml}$ from baseline consumption, respectively). Again, analysis of individual data revealed no significant correlations between changes in saccharin consumption and changes in time spent on the DPS for either of these two conditions, absolute $r \mathrm{~s} \leq .269$, $p$ s $>.05$ (see Table 1).

Experiment 2: morphine, $10 \mathrm{mg} / \mathrm{kg}$

As above, individual subjects injected with morphine $(n=12)$ were divided into high $(n=6)$ and low $(n=6)$

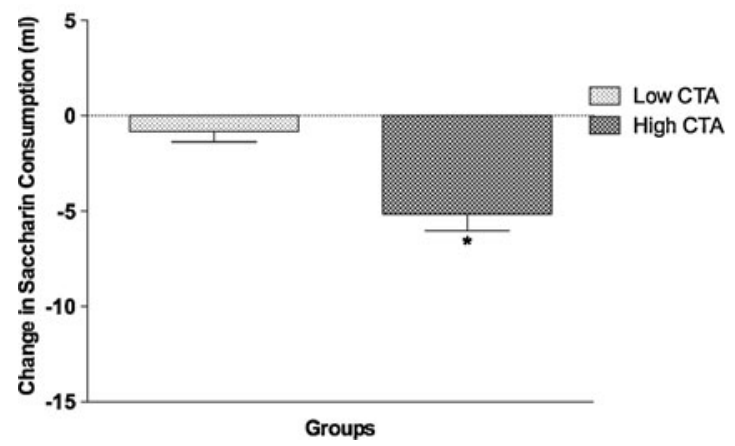

Fig. 1 Change in saccharin consumption (left panel) and change in time spent on the drug-paired side (right panel) for high and low responders in subjects injected with $5 \mathrm{mg} / \mathrm{kg}$ morphine. The high and responders for both the CTA and CPP preparations. Subjects in both the high $(n=6), t(5)=-20.494, p<$ .001 , and low $(n=6), t(5)=-8.283, p<.001$, CTA groups significantly suppressed their saccharin consumption, relative to their own baseline (Fig. 2, left panel). When the high- and low-CTA groups were separately analyzed for place preferences, neither high, $t(5)=0.382, p>.05$, nor low, $t(5)=0.357, p>.05$, CTA groups differed from their respective baseline in time spent on the DPS (13- and 8 -s change from baseline preference, respectively). Analysis of individual subjects revealed no significant correlations between changes in saccharin consumption and changes in time spent on the DPS for either of these two conditions, absolute $r \mathrm{~s} \leq .737, p \mathrm{~s}>.05$ (see Table 1$)$.

The high-CPP group $(n=6), t(5)=4.077, p<.05$, displayed a significant increase in time spent on the DPS, whereas the low-CPP group $(n=6)$ displayed a significant decrease in time spent on the DPS, $t(5)=-3.526, p<.05$ (Fig. 2, right panel). When the high- and low-CPP groups were analyzed for taste aversions, both high, $t(5)=--9.083$, $p<.001$, and low, $t(5)=-5.636, p<0.01$, CPP groups displayed a significant decrease in amount of saccharin consumed ( $-6-$ and $-5-\mathrm{ml}$ change from baseline consumption, respectively). Analysis of individual subjects revealed no significant correlations between changes in saccharin consumption and changes in time spent on the DPS for either of these two conditions, absolute $r s \leq .441, p s>.05$ (see Table 1).

\section{Experiment 3: amphetamine, $3 \mathrm{mg} / \mathrm{kg}$}

When amphetamine-injected subjects $(n=10)$ were divided into high and low responders, subjects in both the high $(n=$ $5), t(4)=-13.023, p<.001$, and low $(n=5), t(4)=$ $-23.717, p<.001$, CTA groups significantly suppressed their saccharin consumption, relative to their own baseline (Fig. 3, left panel). When the high- and low-CTA groups were separately analyzed for place preferences, the high,

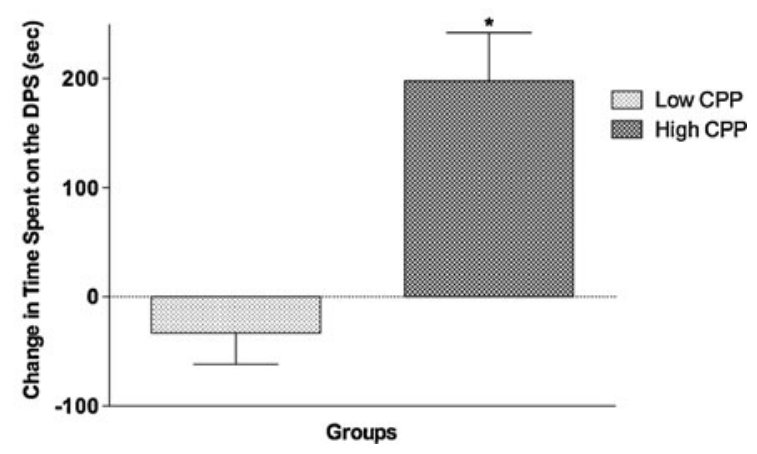

low groups were defined by the median split (see the text for detail). *Significant difference from baseline 
Table 1 Correlational analyses of the relationship between change in time spent on the drug-paired side and change in saccharin consumption for high and low responders across all conditions. Each cell indicated the $r$ and $p$ values for the given relationship. Bold font indicates significant relationship (see text for more detail). Included are also correlational analyses of the relationship for all subjects injected with either both doses of morphine or both doses of amphetamine

\begin{tabular}{lllll}
\hline & High CTA & Low CTA & High CPP & Low CPP \\
\hline Morphine $5 \mathrm{mg} / \mathrm{kg}$ & $r=0.127$ & $r=-0.497$ & $r=0.176$ & $r=-0.269$ \\
& $p=0.811$ & $p=0.316$ & $p=0.738$ & $p=0.607$ \\
Morphine $10 \mathrm{mg} / \mathrm{kg}$ & $r=0.590$ & $r=-0.737$ & $r=-0.441$ & $r=0.210$ \\
& $p=0.218$ & $p=0.095$ & $p=0.381$ & $p=0.690$ \\
Morphine 5 and $10 \mathrm{mg} / \mathrm{kg}$ & $r=0.216$ & $r=0.140$ & $r=0.524$ & $r=-0.121$ \\
& $p=0.499$ & $p=0.663$ & $p=0.081$ & $p=0.709$ \\
Amphetamine 3 $\mathrm{mg} / \mathrm{kg}$ & $r=-0.248$ & $r=0.004$ & $r=0.242$ & $r=0.251$ \\
& $p=0.687$ & $p=0.995$ & $p=0.695$ & $p=0.684$ \\
Amphetamine 5 mg/kg & $\boldsymbol{r}=\mathbf{0 . 8 8 2}$ & $r=-0.543$ & $r=0.589$ & $r=-0.532$ \\
Amphetamine 3 and 5 mg/kg & $\boldsymbol{p}=\mathbf{0 . 0 2 0}$ & $p=0.344$ & $p=0.219$ & $p=0.356$ \\
& $r=-0.335$ & $r=-0.223$ & $r=0.003$ & $r=-0.071$ \\
& $p=0.314$ & $p=0.535$ & $p=0.993$ & $p=0.845$ \\
\hline
\end{tabular}

$t(4)=2.880, p<.05$, but not the low, $t(4)=0.211, p>.05$, CTA group significantly increased their time spent on the DPS (122- and 13-s change from baseline preference, respectively). Analysis of individual subjects revealed no significant relationship between changes in saccharin consumption and changes in time spent on the DPS for either of these two conditions, absolute $r \mathrm{~s} \leq .248, p \mathrm{~s}>.05$ (see Table 1).

The high-CPP group $(n=5), t(4)=12.482, p<.001$, displayed a significant increase in time spent on the DPS, whereas the low-CPP group $(n=5)$ did not differ from its baseline, $t(4)=-1.172, p<.05$ (Fig. 3, right panel). When the high-CPP and low-CPP groups were analyzed for taste aversions, both high, $t(4)=-9.253, p<$ .001 , and low, $t(4)=-9.375, p<.01$, CPP groups displayed a significant decrease in amount of saccharin consumed (-11- and -8-ml change from baseline consumption, respectively). Analysis of individual subjects revealed no significant correlations between changes in saccharin con- sumption and changes in time spent on the DPS for either of these two conditions, absolute $r \mathrm{~s} \leq .251, p \mathrm{~s}>.05$ (see Table 1).

Experiment 4: amphetamine, $5 \mathrm{mg} / \mathrm{kg}$

When amphetamine-injected subjects $(n=11)$ were divided into high and low responders, subjects in both the high $(n=$ $6), t(5)=-26.784, p<.001$, and low $(n=5), t(4)=-12.363$, $p<.001$, CTA groups significantly suppressed their saccharin consumption, relative to their own baseline (Fig. 4, left panel). When the high- and low-CTA groups were separately analyzed for place preferences, neither high, $t(5)=0.716$, $p>.05$, nor low, $t(4)=0.943, p>.05$, CTA groups differed from their respective baseline in time spent on the DPS (21- and 77-s change from baseline preference, respectively). Analysis of individual subjects revealed a significant relationship between change in saccharin consumption and change in time spent on the DPS for individual

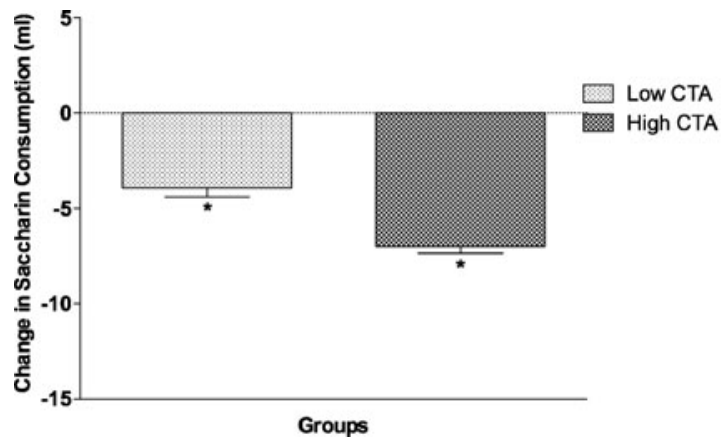

Fig. 2 Change in saccharin consumption (left panel) and change in time spent on the drug-paired side (right panel) for high and low responders in subjects injected with $10 \mathrm{mg} / \mathrm{kg}$ morphine. The high and

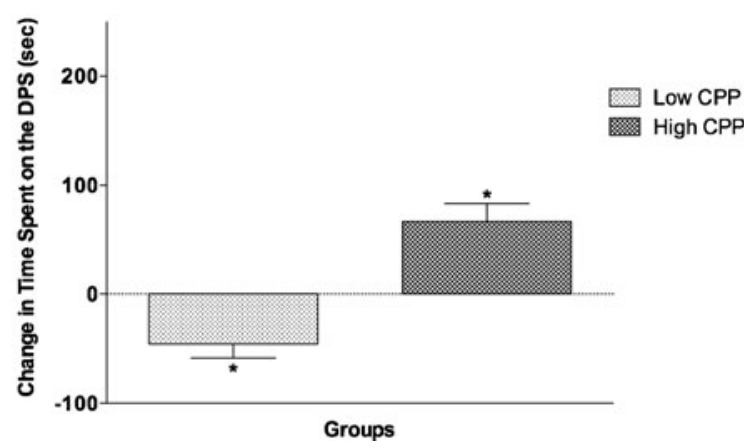

low groups were defined by the median split (see the text for detail). *Significant difference from baseline 


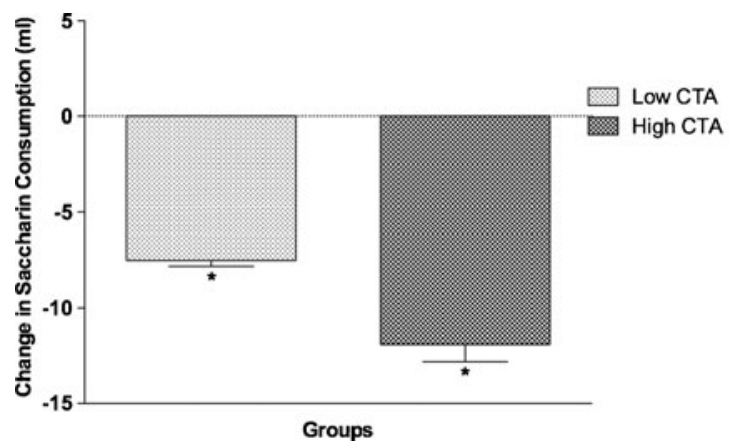

Fig. 3 Change in saccharin consumption (left panel) and change in time spent on the drug-paired side (right panel) for high and low responders in subjects injected with $3 \mathrm{mg} / \mathrm{kg}$ amphetamine. The high

subjects in the high-CTA group, $r=.882, p=.02$. Specifically, animals displaying the greater decrease from baseline in amount of saccharin consumed showed either limited increase (or actual decrease) in time spent on the DPS. There was no significant relationship between change in saccharin consumption and change in time spent on the DPS for individual subjects in the low-CTA group, $r=$ $-.542, p>.05$ (see Table 1).

The high-CPP group $(n=6), t(5)=2.890, p<.05$, displayed a significant increase in time spent on the DPS, whereas the low-CPP group $(n=5)$ displayed a significant decrease in time spent on the DPS, $t(4)=-2.891, p<.05$ (Fig. 4, right panel). When the high-CPP and low-CPP groups were analyzed for taste aversions, both high, $t(5)=-20.656, p<.001$, and low, $t(4)=-7.238, p<.01$, CPP groups displayed a significant decrease in amount of saccharin consumed (-8-ml change from baseline consumption for both groups). Analysis of individual subjects revealed no significant correlations between changes in saccharin consumption and changes in time spent on the DPS for either of these two conditions, absolute $r \mathrm{~s} \leq .589$, $p$ s $>.05$ (see Table 1).

Control subjects in each of the four experiments $(n=10$ 11 per experiment) were also evaluated for changes in saccharin intake and time spent on the white side of the

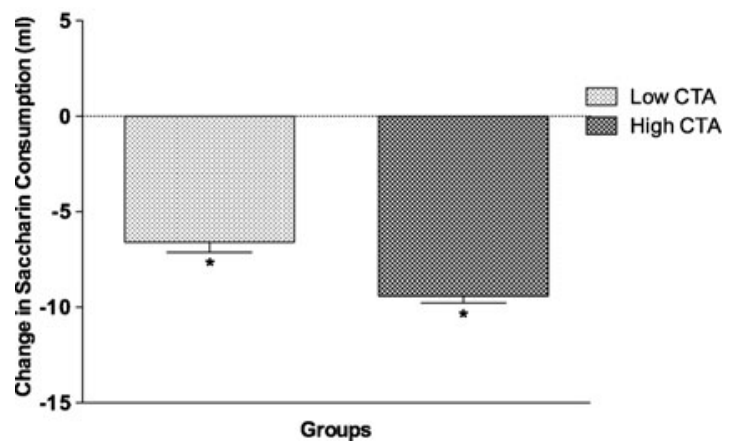

Fig. 4 Change in saccharin consumption (left panel) and change in time spent on the drug-paired side (right panel) for high and low responders in subjects injected with $5 \mathrm{mg} / \mathrm{kg}$ amphetamine. The high

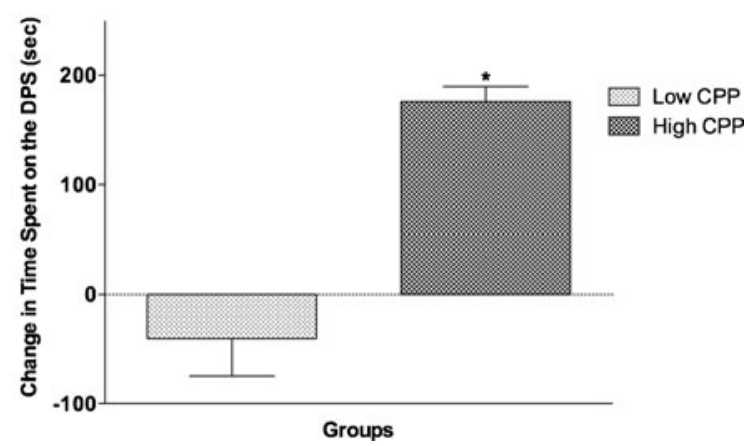

and low groups were defined by the median split (see the text for detail). *Significant difference from baseline

chamber. A paired samples $t$-test analysis revealed a significant increase in amount of saccharin consumed in Experiments 1 and 3,all $t \mathrm{~s} \geq 2.781$, all $p \mathrm{~s}<.05$, but no change in amount of saccharin consumed in Experiments 2 and 4 ,all $t \mathrm{~s} \leq 1.008$, all $p \mathrm{~s}>.05$. The significant increases from baseline consumption in amount of saccharin consumed for subjects in Experiments 1 and 3 likely reflect an initial neophobic response, which has been reported to vary across subjects (see Mitchell, Kirschbaum, \& Perry, 1975). Analysis of the change in time spent on the DPS revealed no significant change for any of the four experiments, absolute $t \mathrm{~s} \leq 1.992$, all $p \mathrm{~s}>.05$.

\section{Discussion}

The reports that preferences and aversions can be conditioned by the same drug are generally used to suggest that drugs of abuse produce co-occurring rewarding and aversive effects (Reicher \& Holman, 1977; Simpson \& Riley, 2005; Switzman et al., 1978; White et al., 1977; Wise et al., 1976; see also Riley et al., 2009). As was noted above, however, the manner by which demonstrations of preferences and aversions are typically generated is by group analysis that precludes any assessment of the covariation of preferences and aversions in

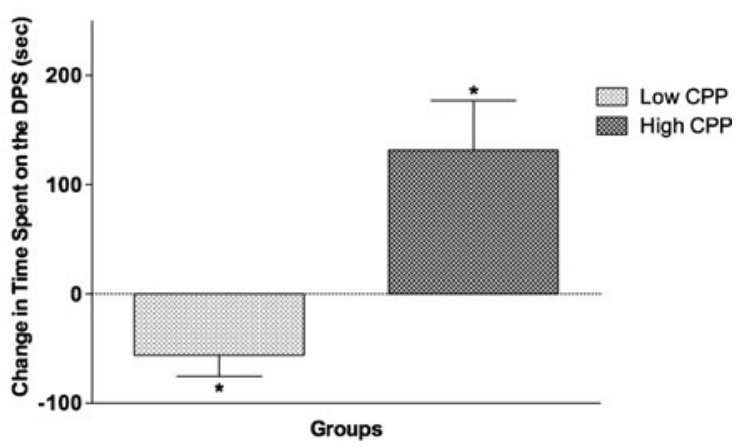

and low groups were defined by the median split (see the text for detail). *Significant difference from baseline 
individual subjects. If these effects covary within individual subjects in a dependent manner (i.e., relationship between the two), this would strongly argue for a commonality of the underlying mechanisms mediating these effects. If, on the other hand, there is no relationship between the ability of a drug to produce place preferences and taste aversions within individual subjects, these opposing motivational effects are likely mediated by different mechanisms. To more directly address this issue, the present experiments examined the relationship between the ability of morphine and amphetamine to condition place preferences and taste aversions in individual subjects.

As has been described, rats were given one tastedrug-environment pairing with either a low or high dose of morphine ( 5 and $10 \mathrm{mg} / \mathrm{kg}$ ) or a low or high dose of amphetamine ( 3 and $5 \mathrm{mg} / \mathrm{kg}$ ) or were injected with vehicle. Place preferences and taste aversions were analyzed in high and low responders to assess the ability of either drug and at either dose to condition preferences and aversions. When place preferences and taste aversions were analyzed in high and low responders across all four experiments, subjects in high-CPP and high-CTA groups always showed significant preferences and aversions across different drugs and different doses. The performance of low responders was less consistent with subjects in the low-CPP groups, showing either no change from baseline in time spent on the DPS $(5 \mathrm{mg} /$ $\mathrm{kg}$ morphine and $3 \mathrm{mg} / \mathrm{kg}$ amphetamine) or an actual decrease in time spent on the DPS $(10 \mathrm{mg} / \mathrm{kg}$ morphine and $5 \mathrm{mg} / \mathrm{kg}$ amphetamine). Subjects in the low-CTA groups showed significant aversions at all but the low dose of morphine $(5 \mathrm{mg} / \mathrm{kg})$.

Although preferences and aversions were established in the specific group analyses (e.g., high CPP, high CTA), there was no consistent relationship between reward and aversion in individual subjects. Of the 16 possible correlations generated, there was only 1 , that is, Group High CTA in the $5-\mathrm{mg} / \mathrm{kg}$ amphetamine group, for which the relationship was significant. Under this condition, subjects that showed the greater decrease in amount of saccharin consumed were more likely to display either a limited increase or an actual decrease in time spent on the DPS-interestingly, a relationship inconsistent with the position that a common mechanism underlies the two behaviors (see below).

This dissociation between place preferences and taste aversions in individual subjects does not argue that drugs do not produce both effects. They clearly do, as evidenced by studies that have examined preferences and aversions between and within groups (see above). The present data do argue, however, that the rewarding and aversive effects may be independent, with individual animals displaying varying degrees of each (possibly due to differential reactivity for each effect). If they were dependent upon each other, an animal would be expected to respond comparably-for example, display both high CTA and high CPP or low CTA and low CPP. The lack of a significant relationship is inconsistent with this position and argues that the rewarding and aversive properties of a drug are independent.

The present series of experiments are the first to compare concurrently place preferences and taste aversions in individual subjects. As was noted above, other studies generally have examined aversions in separate groups of subjects (within or across studies), precluding individualsubject analysis. Only a single study (Turenne et al., 1996) has examined the possible relationship between aversions and preferences in the same subjects, but as was noted, it did not analyze individual-subject data and used a serial taste/place conditioning procedure (see above). Although the present series of assessments and those of Turenne et al. do differ procedurally and in focus, comparisons can be made, albeit cautiously. In relation to morphine, the present results are consistent with those of Turenne et al. in that there was no apparent relationship between the ability of the drug to induce preferences and aversions. Our results with amphetamine, however, differ from those of Turenne et al. More specifically, Turenne et al. reported that the group that displayed the strongest amphetamine-induced CTA also showed the strongest amphetamine-induced CPP; in the present experiments with amphetamine, we found a relationship only with animals conditioned with the highest dose of amphetamine, and under this condition, the relationship was opposite to that reported by Turenne et al. Specifically, subjects that showed the greater decrease in amount of saccharin consumed were less likely to display a place preference (as indexed by time spent on the DPS). No other correlational analyses revealed a significant relationship between amphetamine-induced reward and aversion.

There are several important differences between our study and theirs that may account for the reported differences with amphetamine. One such difference is that in Turenne et al. (1996), subjects received taste and place conditioning in a serial manner, while in the present studies, animals received concurrent CTA/CPP conditioning. Another difference in the two reports is that in Turenne et al., animals received different number of conditioning trials for both taste aversion and place preference conditioning. Thus, animals injected with amphetamine received a single taste aversion conditioning trial and two, four, or eight trials in place preference conditioning. In the present series of experiments, subjects were equated in the number of conditioning trials across all experiments and received a single CTA/CPP conditioning trial. Moreover, although both studies used the same doses of amphetamine ( 3 and $5 \mathrm{mg} / \mathrm{kg}$ ) given by the same route of administration, Turenne et al. pooled and analyzed the data across the 
doses, whereas in the present report, the doses were analyzed in separate experiments. However, when the data from the two morphine and two amphetamine conditions were pooled, we still found no relationship between the ability of either drug to condition place preference and taste aversion (see more details below). It is not known to what extent these factors contributed to the differences in the two reports. It is clear, however, that when the rewarding and aversive effects of morphine and amphetamine are examined in individual subjects (vs. group analysis in all other studies), we see no consistent relationship between the ability of either drug to produce these effects concurrently in the same animal.

Although there was no clear relationship in the present series of experiments between the ability of morphine or amphetamine to increase time spent on the DPS and to suppress saccharin consumption, there are several caveats that deserve discussion. First, the size for each group of high and low responders was small (5-6 subjects per group), which may limit our conclusions regarding the absence of relationships. It should be noted, however, that when the individual-subject data from the two morphine and two amphetamine doses were pooled and analyzed together (see Turenne et al., 1996), there still was no relationship between reward and aversion (see Table 1). In other words, when the $n$ was functionally doubled for either drug condition, we still found no relationship between the two.

Second, although CPP and CTA are thought to measure the rewarding and aversive effects of drugs, respectively, these procedures may differ in their relative sensitivity as such measures. For example, CPP may be less sensitive as a measure of the rewarding effects of morphine than CTA is as a measure of the aversive effects of the drug. As such, the rewarding effects of morphine in the present study may not have been accurately reflected in the expression of place preferences for specific animals. Conversely, CTA may be less sensitive as a measure of the aversive effects of morphine than CPP is as a measure of the rewarding effects of the drug, and the aversive effects of morphine in the present study may not have been accurately reflected in the expression of taste aversions for specific animals. As such, any attempt to relate these behaviors in a correlational analysis should be made cautiously. However, when the relationship between change in time spent on the DPS and change in saccharin consumption was examined in subjects showing either significant preferences (i.e., high CPP) or significant aversions (i.e., high CTA), there still was no consistent significant correlation between the two. The only exception to this was for the high-CTA group given the $5-\mathrm{mg} / \mathrm{kg}$ amphetamine dose. In other words, under conditions where preferences and aversions were analyzed in individual subjects most sensitive to either rewarding (high CPP) or aversive (high CTA) effects of either drug and at either dose, there still was no consistent relationship between the ability of either drug to produce these effects (see Table 1).

Third, although preferences and aversions were clearly acquired, the present study does not address the nature of these effects - that is, the specific stimulus effects of morphine or amphetamine responsible for this conditioning. For example, in relation to morphine's rewarding effects, it remains unknown whether morphine supports a place preference due to some euphoric, positive effect or the negatively rewarding effect of anxiety reduction (see van der Kooy, 1987). Although one generally assumes that such preferences reflect a positive effect of the drug, in biased designs, such as the one used here, in which animals are placed in the initially nonpreferred compartment following drug injection, increases in time on the DPScould be due to the anxiolytic effects of the drug. How this would apply to amphetamine, however, is unclear. Similarly, the basis for taste aversion learning, for both morphine and amphetamine, remains a subject of considerable debate. While some have argued that aversions are based on sickness or a dysphoric effect of the drug (Riley \& Tuck, 1985), others have noted that drug novelty or disruption of homeostasis mediates taste aversions (Gamzu, 1977; Hunt \& Amit, 1987). Yet others have argued that aversions misrepresent the suppression of consumption evident in such designs and that taste avoidance better characterizes the suppression (Parker, 1988, 1991, 1995).

Independently of the specific bases for these two behavioral effects (i.e., place preferences and taste aversions), it is generally assumed that they reflect different motivational or affective properties of drugs of abuse (Riley et al., 2009). The present results are consistent with this assumption in that the rewarding and aversive effects of morphine and amphetamine appear dissociated. This dissociation has implications for any interpretation of taste aversion learning that argues that the rewarding and aversive effects of drugs of abuse are mediated by a common mechanism. For example, Grigson (1997) has argued that a drug's well-documented rewarding, rather than its hypothetical aversive, effects mediate suppression of saccharin consumption in the CTA design. According to this position, rats avoid a rewarding saccharin solution in anticipation of a more rewarding drug (i.e., reward comparison). This hypothesis, however, fails to explain the findings of the present study in which the rewarding and suppressive effects of morphine and amphetamine appear dissociated. The only exception in the present analyses was observed in the high CTA in the $5 \mathrm{mg} / \mathrm{kg}$ amphetamine group and is inconsistent with the position that the rewarding effects of drugs mediate aversion learning (subjects that showed the greater 
decrease in amount of saccharin consumed were less likely to display a place preference). The present results, on the other hand, are consistent with the position that drugs of abuse are complex pharmacological stimuli that have multiple stimulus effects, both rewarding and aversive, that condition place preference and taste aversion separately. Moreover, as described above, the occurrence of one effect does not necessarily predict the occurrence (or the extent) of the other.

It has been argued that the initiation and/or escalation of drug taking may be a function of the relative balance between the rewarding and aversive effects of drugs of abuse (Gaiardi et al., 1991; Riley et al., 2009; Riley \& Simpson, 2001). As such, the ongoing effort to examine different factors that may impact susceptibility to drug use (e.g., drug self-administration) warrants a better understanding of the relationship between a drug's rewarding and aversive effects. The present findings show significant individual variability in response to the affective properties (both rewarding and aversive) of morphine and amphetamine. Given this considerable variability, it is important to further examine the contribution of this individual variability to the vulnerability to drug self-administration.

\section{References}

Allen, R. M., Everett, C. V., Nelson, A. M., Gulley, J. M., \& Zahniser, N. R. (2007). Low and high locomotor responsiveness to cocaine predicts intravenous cocaine conditioned place preference in male Sprague-Dawley rats. Pharmacology Biochemistry and Behavior, 86, 37-44.

Brockwell, N. T., Eikelboom, R., \& Beninger, R. J. (1991). Caffeineinduced place and taste conditioning: production of dosedependent preference and aversion. Pharmacology Biochemistry and Behavior, 38, 513-517.

Cunningham, C. L., Gremel, C. M., \& Groblewski, P. A. (2009). Genetic influences on conditioned taste aversion. In S. Reilly \& T. R. Schachtman (Eds.), Conditioned taste aversion: Behavioral and neural processes (pp. 387-421). New York: Oxford University Press.

Farber, P. D., Gorman, J. E., \& Reid, L. D. (1976). Morphine injections in the taste aversion paradigm. Physiological Psychology, 4, 265-268.

Gaiardi, M., Bartoletti, M., Bacchi, A., Gubellini, C., Costa, M., \& Babbini, M. (1991). Role of repeated exposure to morphine in determining its affective properties: place and taste conditioning studies in rats. Psychopharmacology, 103, 183-186.

Gamzu, E. (1977). The multifaceted nature of taste-aversioninducing agents: Is there a single common factor? In L. M. Barker, M. R. Best \& M. Domjan (Eds.), Learning mechanisms in food selection (pp. 477-510). Waco: Baylor University Press.

Gomez-Serrano, M. A., Kearns, D. N., \& Riley, A. L. (2009). The effects of light cycle phase on morphine-induced conditioned taste aversions in the Lewis, Fischer and Sprague-Dawley rat strains. Behavioural Brain Research, 196, 116-122.
Grigson, P. S. (1997). Conditioned taste aversions and drugs of abuse: a reinterpretation. Behavioral Neuroscience, 111, 129136.

Hunt, T., \& Amit, Z. (1987). Conditioned taste aversion induced by self-administered drugs: paradox revisited. Neuroscience and Biobehavioral Review, 11, 107-130.

Martin, G. M., Bechara, A., \& van der Kooy, D. (1988). Morphine preexposure attenuates the aversive properties of opiates without preexposure to the aversive properties. Pharmacology Biochemistry and Behavior, 30, 687-692.

Mitchell, D., Kirschbaum, E. H., \& Perry, R. L. (1975). Effects of neophobia and habituation on the poison-induced avoidance of exteroceptive stimuli in the rat. Journal of Experimental Psychology, 104, 47-55.

National Research Council. (1996). Guide for the care and use of laboratory animals. Washington: National Academy Press.

Parker, L. A. (1988). Positively reinforcing drugs may produce a different kind of CTA than drugs which are not positively reinforcing. Learning and Motivation, 19, 207-220.

Parker, L. A. (1991). Taste reactivity responses elicited by reinforcing drugs: A dose-response analysis. Behavioral Neuroscience, 105, 955-964.

Parker, L. A. (1995). Rewarding drugs produce taste avoidance, but not taste aversion. Neuroscience and Biobehavioral Review, 19, $143-157$.

Parker, L. A., Limebeer, C. L., \& Rana, S. A. (2009). Conditioned disgust, but not conditioned taste avoidance, may reflect conditioned nausea in rats. In S. Reilly \& T. R. Schachtman (Eds.), Conditioned taste aversion: Behavioral and neural processes (pp. 92-113). New York: Oxford University Press.

Randall-Thompson, J. F., \& Riley, A. L. (2003). Morphine-induced conditioned taste aversions: assessment of sexual dimorphism. Pharmacology Biochemistry and Behavior, 76, 373-381.

Reicher, M. A., \& Holman, E. W. (1977). Location preference and flavor aversion reinforced by amphetamine in rats. Animal Learning \& Behavior, 5, 343-346.

Riley, A. L., Davis, C. M., \& Roma, P. G. (2009). Strain differences in taste aversion learning: implications for animal models of drug abuse. In S. Reilly \& T. R. Schachtman (Eds.), Conditioned taste aversion: Behavioral and neural processes (pp. 226-261). New York: Oxford University Press.

Riley, A. L., Jacobs, W. J., \& LoLordo, V. M. (1978). Morphineinduced taste aversions: A consideration of parameters. Physiological Psychology, 6, 96-100.

Riley, A. L., \& Simpson, G. R. (2001). The attenuating effects of drug preexposure on taste aversion conditioning: Generality, experimental parameters, underlying mechanisms, and implications for drug use and abuse. In R. R. Mowrer \& S. B. Klein (Eds.), Handbook of contemporary learning theories (pp. 505-559). Mahwah: Erlbaum.

Riley, A. L., \& Tuck, D. L. (1985). Conditioned taste aversions: a behavioral index of toxicity. Annals of the New York Academy of Sciences, 443, 272-292.

Sherman, J. E., Pickman, C., Rice, A., Liebeskind, J. C., \& Holman, E. W. (1980). Rewarding and aversive effects of morphine: Temporal and pharmacological properties. Pharmacology Biochemistry and Behavior, 13, 501-505.

Simpson, G. R., \& Riley, A. L. (2005). Morphine preexposure facilitates morphine place preference and attenuates morphine taste aversion. Pharmacology Biochemistry and Behavior, 80, 471-479.

Switzman, L., Amit, Z., White, N., \& Fishman, B. (1978). Noveltasting food enhances morphine discriminability in rats. In F. C. Colpaert \& J. A. Rosecrans (Eds.), Stimulus properties of drugs: Ten years of progress (pp. 199-207). Amsterdam: Elsevier/NorthHolland Biomedical Press. 
Turenne, S. D., Miles, C., Parker, L. A., \& Siegel, S. (1996). Individual differences in reactivity to the rewarding/aversive properties of drugs: assessment by taste and place conditioning. Pharmacology Biochemistry and Behavior, 53, 511516.

Tzschentke, T. M. (2007). Measuring reward with the conditioned place preference (CPP) paradigm: update of the last decade. Addiction Biology, 12, 227-462.

van der Kooy, D. (1987). Place conditioning: a simple and effective method for assessing the motivational properties of drug. In M. A. Bozarth (Ed.), Methods of assessing the reinforcing properties of abused drugs (pp. 229-240). New York: Springer.

White, N., Sklar, L., \& Amit, Z. (1977). The reinforcing action of morphine and its paradoxical side effect. Psychopharmacology, 52, 63-66.

Wise, R. A., Yokel, R. A., \& DeWit, H. (1976). Both positive reinforcement and conditioned aversion from amphetamine and from apomorphine in rats. Science, 191, 1273-1275.

$\mathrm{Xu}$, N., Wang, L., Wu, C., \& Pei, G. (2001). Spatial learning and morphine-rewarded place preference negatively correlates in mice. Pharmacology Biochemistry and Behavior, 68, 389-394. 